\title{
Clinical and videofluoroscopic diagnosis of dysphagia in chronic encephalopathy of childhood
}

\author{
Avaliação clínica e videofluoroscopia no diagnóstico de disfagia na encefalopatia crônica da infância
}

Brenda Carla Lima Araújoํㅜㄹ Maria Eugênia Almeida Motta ${ }^{2}$, Adriana Guerra de Castro ${ }^{3}$, Claudia Marina Tavares de Araújo ${ }^{4}$

Araújo BCL, Motta MEA, Castro AG, Araújo CMT. Clinical and videofluoroscopic diagnosis of dysphagia in chronic encephalopathy of childhood. Radiol Bras. 2014 Mar/Abr;47(2):84-88.

Abstract Objective: To evaluate the contribution of deglutition videofluoroscopy in the clinical diagnosis of dysphagia in chronic encephalopathy of childhood.

Materials and Methods: The study sample consisted of 93 children diagnosed with chronic encephalopathy, in the age range between two and five years, selected by convenience among patients referred to the authors' institution by speech therapists, neurologists and gastroenterologists in the period from March 2010 to September 2011. The data collection was made at two different moments, by different investigators who were blind to each other.

Results: The method presented low sensitivity for detecting aspiration with puree consistency $(p=0.04)$. Specificity and negative predictive value were high for clinical diagnosis of dysphagia with puree consistency.

Conclusion: In the present study, the value for sensitivity in the clinical diagnosis of dysphagia demonstrates that this diagnostic procedure may not detect any change in the swallowing process regardless of the food consistency used during the investigation. Thus, the addition of the videofluoroscopic method can significantly contribute to the diagnosis of dysphagia.

Keywords: Swallowing disorders; Videofluoroscopy; Clinical diagnosis; Sensitivity; Specificity.

Resumo Objetivo: Avaliar se o diagnóstico clínico da disfagia foi potencializado pelo método videofluoroscópico na encefalopatia crônica da infância.

Materiais e Métodos: A amostra foi constituída por 93 crianças com diagnóstico de encefalopatia crônica da infância, com idade entre dois e cinco anos, selecionadas por conveniência, de encaminhamentos vindos de fonoaudiólogos, neuropediatras e gastrenterologistas no período de março de 2010 a setembro de 2011. A coleta se deu em dois momentos distintos, com pesquisadores diferentes e cegos entre si.

Resultados: Foi observada baixa sensibilidade para detectar a aspiração na consistência pastosa $(p=0,04)$. Os valores de especificidade e valor preditivo negativo foram altos para diagnóstico clínico da disfagia na consistência pastosa.

Conclusão: Neste estudo, o valor da sensibilidade do diagnóstico clínico da disfagia demonstra que este procedimento de diagnóstico pode não detectar comportamentos alterados no processo de deglutição, independente da consistência utilizada durante a investigação. Desta forma, o diagnóstico clínico da disfagia foi potencializado pelo método videofluoroscópico.

Unitermos: Transtornos de deglutição; Videofluoroscopia; Diagnóstico clínico; Sensibilidade; Especificidade.

\section{INTRODUCTION}

Chronic encephalopathy of childhood is characterized by various motor and postural impairments resulting from the neurological involvement. Such conditions may affect oral motor structures, leading to feeding difficulties result-

* Study developed in the Department of Post-Graduation in Child and Adolescent Health at Universidade Federal de Pernambuco (UFPE), Recife, PE, Brazil. Financial support: Fundação de Amparo à Ciência e Tecnologia do Estado de Pernambuco (Facepe) and Conselho Nacional de Desenvolvimento Científico e Tecnológico (CNPq).

1. Master, Assistant Professor, Department of Speech and Language Pathology Department, Universidade Federal de Sergipe (UFS), São Cristóvão, PE, Brazil.

2. PhD, Professor, Maternal-Infantile Department, Universidade Federal de Pernambuco (UFPE), Recife, PE, Brazil.

3. PhD, Clinical Speech Therapist, Recife, PE, Brazil.

4. PhD, Professor, Department of Phonoaudiology, Universidade Federal de Pernambuco (UFPE), Recife, PE, Brazil. ing in impairment of the transit of food from the oral cavity to the stomach, which characterizes the presence of dysph$\operatorname{agia}^{(1-5)}$

The diagnosis of dysphagia is achieved by means of clinical and instrumental evaluation, both fundamentally important. Such investigations are based on subjective and objective parameters in the characterization and differentiation between normal and altered behaviors. Thus, what one observes in the practice of clinical investigations is the need for and increasingly more accurate diagnosis of dysphagia, in the search for information relevant for the therapeutic process in children with feeding difficulties.

Mailing Address: Dra. Brenda Carla Lima Araújo. Avenida Governador Carlos de Lima Cavalcanti, 3037, ap. 703, Casa Caiada. Olinda, PE, Brazil, 53130-530. E-mail: brendaaraujo@yahoo.com.br.

Received February 21, 2013. Accepted after revision October 17, 2013. 
The clinical diagnosis of swallowing disorders comprises the observation of several components of the feeding process, such as the oral motor functions, posture, tonus and mobility of structures involved in the orofacial complex ${ }^{(6)}$. Such an evaluation is important for the definition of recommendations for safe feeding, without risks of aspiration, besides the establishment of rehabilitation strategies.

However, an aspect that is considered as being fragile in the clinical diagnosis, is the lack of objectivity, sometimes failing to detect altered patterns. In other words, this type of evaluation may fail to accurately identify changes in any of the phases that comprise deglutition dynamics ${ }^{(6-9)}$.

Such an assertion reflects situations where the clinical diagnosis may generate doubts on the presence of food and/ or saliva aspiration. Thus it is probable that, on many occasions, the health professional will need to investigate such behaviors by indicating the most appropriate complementary method to evaluate each specific clinical condition.

The videofluoroscopy emerges as a complementary diagnostic method in cases of dysphagia, since it can provide real time images of all swallowing phases, allowing for the dynamic analysis of this function ${ }^{(10)}$. Such a method is considered the gold standard for the investigation of aspiration. Some studies highlight the importance of this method in the diagnosis and treatment of dysphagia in the pediatric population $^{(11,12)}$.

Thus, one observes the importance of defining whether the clinical diagnosis of dysphagia might be enhanced by further investigation, as this is a fundamental practice in order to ensure the clinical diagnosis accuracy.

The present study was aimed at evaluating the sensitivity, specificity, positive and negative predictive values of the clinical diagnosis of dysphagia in children presenting with chronic encephalopathy as compared with the videofluoroscopic method.

\section{MATERIALS AND METHODS}

\section{Patients}

The study sample included 93 children ( 2 to 5 years of age; $60.2 \%$ boys) diagnosed with chronic encephalopathy, considering the presence of cerebral palsy, neuropsychomotor retardation and/or neuromotor disfunction, regardless of severity. The patients were selected at Hospital das Clínicas - Universidade Federal de Pernambuco (UFPE). The presence of orofacial and/or gastric malformations was considered as exclusion criteria. The investigation was duly approved by the Committee for Ethics in Research of the Health Sciences Center of UFPE under No. 108/2011. All caretakers were explained about the investigation and signed a term of free and informed consent.

\section{Procedures}

\section{Clinical evaluation of swallow}

The swallow phases were clinically evaluated by means of a standardized protocol, during swallowing of puree and liquid foods. At that moment, cervical auscultation was also performed, utilizing a stainless steel neonatal stethoscope (Mikatos ${ }^{\circledR}$; São Paulo, Brazil) positioned on one of the lateral aspects of the thyroid cartilage. The different consistencies of food were offered by the caretakers, who were instructed to do it in the same way they did at home, in an attempt to reproduce the caretaker's and child's routine. The observation of feeding was carried out by means of the standardized offering of $50 \mathrm{ml}$ of orange juice $+5 \mathrm{~g}$ of instant food thickener for puree consistency and $50 \mathrm{ml}$ of industrialized juice for liquid consistency.

The foods were offered in spoons and in plastic cups, for the pureed consistency and liquid consistency, respectively. On the course of the evaluation and once it was completed, events suggesting clinical signs of laryngeal penetration and/or aspiration, such as crying, coughing, choking, vomiting, drowsiness, dyspnea and changes in vocal quality, were recorded. Clinical evaluations followed the sequence of pureed and liquid food intake and were performed by two speech therapists trained in the Bobath Neuro-developmental Concept, both with more than 10-year experience in evaluation and rehabilitation of children with neurological alterations.

\section{Videofluoroscopic evaluation of swallow}

The videofluoroscopy ${ }^{(11)}$ was performed according to standardized protocol utilizing a remotely commanded seriograph (VMI; Serimatic Pulsar Plus ${ }^{\circledR}$, Lagoa Santa, Brazil), with the table tilted to $90^{\circ(13,14)}$. Lateral radiological view was utilized, as it better shows the airways patency ${ }^{(15)}$. The focus of the lateral fluoroscopic image was delimited as follows: 1) anterior region, by the lips; 2) superior region,

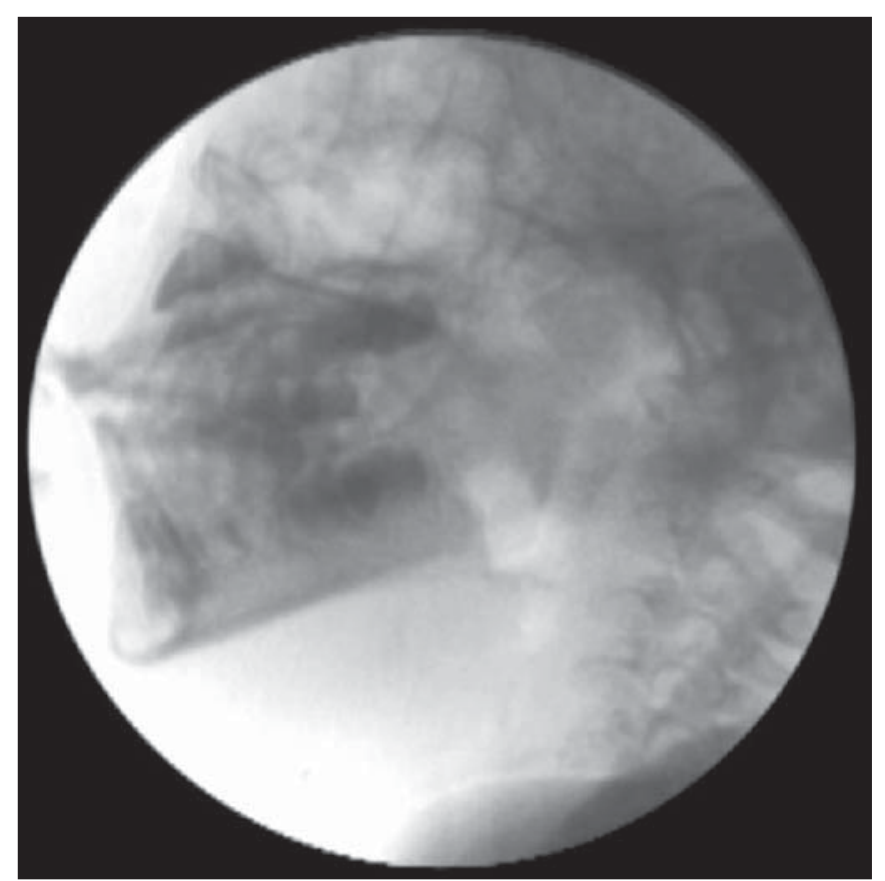

Figure 1. Fluoroscopic image of a child with chronic encephalopathy of childhood. 


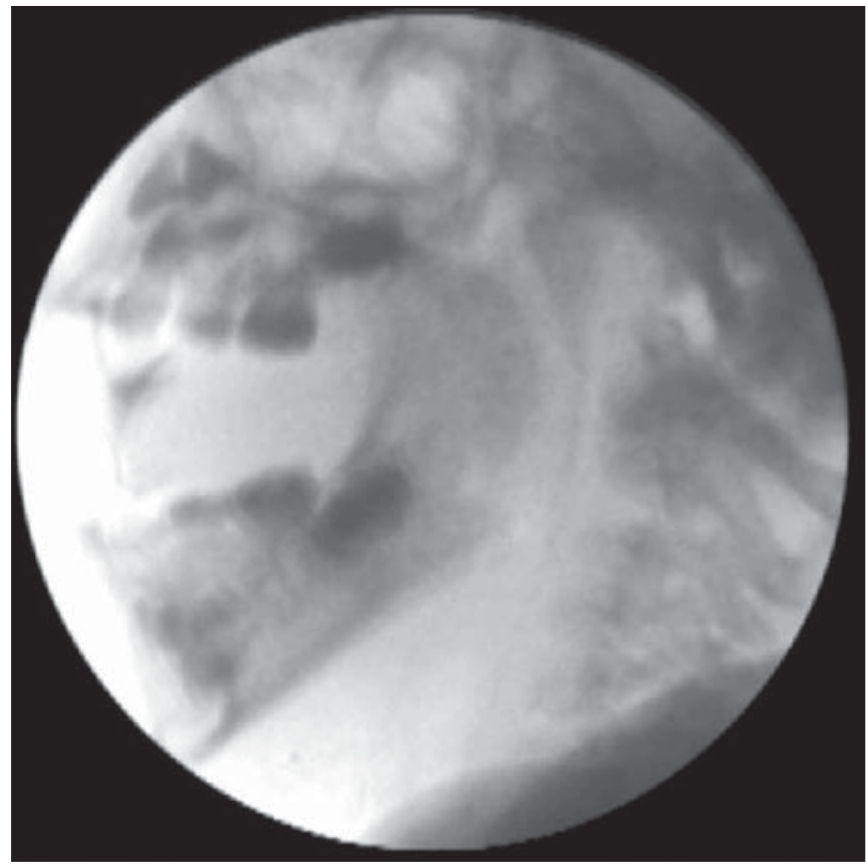

Figure 2. Fluoroscopic image acquired at the beginning of deglutition in a child with chronic encephalopathy of childhood.

by the nasal cavity; 3 ) posterior region, by the cervical spine; 4) inferior region, by the bifurcation of the airway and cervical esophagus $^{(16)}$.

The images were transmitted to a 14-inch double display and, simultaneously, to a video recorder (model RH397H; LG ${ }^{\circledR}$, Manaus, Brazil), where the images were transferred to a DVD. The food was offered by the caretaker to the child. The consistency of the foods and the utilized utensils were equivalent to those utilized in the clinical evaluation. Additionally, $10 \mathrm{ml}$ barium sulfate $(1 \mathrm{~g} / \mathrm{ml})$ was added as contrast agent, as indicated for fluoroscopy studies of the upper digestive tract. All the studies were performed by a single specialized speech therapist with seven-year experience, and by a radiologist. A video (http://youtu.be/8Dlh3aeOZno), performed with the child on right lateral view, demonstrates the difficulties in the oral and pharyngeal phases of deglutition in chronic encephalopathy, which may characterize changes in feeding.

The two collection times occurred at different moments and with different investigators, blinded to each other, i.e., the investigator who performed swallow fluoroscopy was not informed on the performance of the child in the clinical evaluation and vice-versa. The time interval between clinical evaluation and the videofluoroscopy ranged between 7 and 20 days, according to the child availability.

The aspiration parameters (entrance of material beyond the laryngeal ventricle, reaching the lower airway, occurring before, during or after swallowing, either with or without the presence of protective coughing) were evaluated with the pureed and liquid consistencies. Dysphagia was defined by the presence of aspiration both at clinical evaluation and at videofluoroscopy.

\section{Statistical analysis and data processing}

The study was developed under the guidance of the STARD checklist $^{(17)}$. For the statistical analysis, the chi-squared test was applied, utilizing the Statistical Package for the Social Sciences 13.0 (SPSS for Windows) software, considering the value of $p<5 \%$ as statistical significant. The following calculations were performed: sensitivity values (identification of true positive results), specificity (identification of true negative results), positive predictive value (proportion of true positive results in relation to the individuals with positive tests results), and negative predictive value (proportion of true negative results in relation to the individuals with true negative tests results). The results are presented on a $2 \times 2$ table with respective absolute and relative frequencies.

\section{RESULTS}

At the initial clinical evaluation, the authors observed that $43.0 \%$ of the children demonstrated verbal comprehension, $53.5 \%$ interacted with some form of communication, and $52.7 \%$ presented with stridor. As regards feeding, $49.5 \%$ were exclusively mouth fed, with presence of choking during feeding, $95.7 \%$ had predominantly pureed foods in their diets, and $68.8 \%$ ate their meals or were fed sitting on an adult's lap.

Table 1 presents sensitivity, specificity and predictive values for aspiration with pureed consistency foods, adopting videofluoroscopy as the gold standard.

The authors observed that the clinical evaluation presented low sensitivity for detecting aspiration in the evaluated food consistency, and found a statistically significant relationship between the variables $(p=0.04)$.

It is important to mention that it was not possible to observe aspiration in the clinical evaluation for liquid consistency in five of the children, and for pureed consistency in one child. This happened because, during the exams, offering the puree consistency food first and liquid consistency afterwards, was assumed as a standard.

Thus, any event, such as aspiration, with the pureed consistency food (the firstly offered food) led to interruption of the procedure in order to avoid more food entering the respiratory tract.

\section{DISCUSSION}

The present study results have demonstrated low sensitivity of the clinical diagnosis of dysphagia as compared with

Table 1-Sensitivity, specificity and predictive values for clinical diagnosis of aspiration with pureed consistency foods.

\begin{tabular}{lcccccc}
\hline & \multicolumn{4}{c}{$\begin{array}{c}\text { Aspiration (pure food) } \\
\text { - videofluoroscopy }\end{array}$} \\
\cline { 2 - 4 } $\begin{array}{l}\text { Aspiration (pured } \\
\text { food) - clinical } \\
\text { evaluation }\end{array}$ & \multicolumn{2}{c}{ Yes } & & \multicolumn{2}{c}{ No } & \\
\cline { 2 - 4 } \cline { 5 - 6 } & $n$ & $\%$ & & $n$ & $\%$ & p-value* \\
\hline Yes & 4 & 16.7 & 2 & 3.0 & 0.04 \\
No & 20 & 83.3 & 65 & 97.0 & \\
\hline
\end{tabular}

* Exact Fisher's test. Sensitivity $=16 \%$; Positive predictive value $=66 \%$; Specificity $=97 \%$; Positive predictive value $=76 \%$. 
videofluoroscopy with pureed consistency foods in the studied sample. On the other hand, high specificity and high negative predictive value were observed in for the clinical diagnosis with the same food consistency. Such findings indicate that that the accurate clinical diagnosis of dysphagia may not be sufficient in some cases, i.e., probably swallow dysfunctions are not being timely identified and approached, increasing the risk of complications. Similar findings were observed by other authors ${ }^{(11,18)}$ who have concluded that the clinical diagnostic evaluation may fail to detect some difficulties in the swallow process. However, it is observed that a robust methodology with consistent techniques and appropriate standardization of the food consistencies was not utilized in such studies.

The fact that the specificity value was high may suggest that the clinical diagnostic evaluation of the study population detected children with normal swallow patterns, i.e., those children with low or no risk for saliva and/or food aspiration. Such finding is relevant considering that, for the purpose of diagnosing dysphagia, such method should be more sensitive than specific, with a satisfactory positive predictive value, since the failure in identifying children presenting with risk for aspiration and specific difficulties in swallow would bring functional impairment to the feeding process, repetition pneumonia and weight loss in addition to less effective outcomes in swallow rehabilitation.

Studies involving children with chronic encephalopathy demonstrate major impairment in swallowing for liquid consistency $^{(2,19,20)}$. It is possible that such a fact is justifiable because the dysphagia with liquid consistency is more easily diagnosed at clinical evaluation. On the other hand, the failure to identify children presenting with risk for aspiration and/or feeding difficulties may lead to repetition pneumonias and weight loss besides confusing the professional on his thoughts about efficient therapeutic approaches.

An important point that must be emphasized is that, in spite of the fact that some of the mentioned studies were not undertaken exclusively with children or with a population similar to that in the present study, the clinical evaluation should be aimed at diagnosing changes in deglutition, particularly in those cases involving risk for aspiration, regardless of the exposed individual or baseline disease. The present study results suggest that children with deglutition disorders may not be accurately diagnosed by means of clinical evaluation as a single diagnostic method. Thus, the consequences of such practice suggest less than appropriate therapeutic approaches, as a correct, accurate and detailed diagnosis can be instrumental in the rehabilitation process ${ }^{(21,22)}$. One should highlight that the clinical diagnosis is extremely important for therapy, mainly for being an active instrument in the management of children with swallow changes.

Perhaps for such reasons, most authors agree that the clinical diagnosis of dysphagia and videofluoroscopy are complementary diagnostic tools, and in spite of evaluating a single event, different aspects are evaluated, namely, form (technique of the investigator, instruments and patients' life routines) and moment of the evaluation (different days, thus the clinical manifestations of encephalopathy may possibly affect the general conditions of the patient, making him/her more or less predisposed to the manipulation inherent to the evaluations $)^{(2,23,24)}$. Therefore, depending upon the degree of swallow impairment, the accurate clinical diagnosis becomes difficult, requiring a more objective evaluation. The videofluoroscopy is a dynamic and quantifying imaging method, as it allows for the visualization of the entire swallow process, being useful in the diagnostic evaluation of such patients $^{(6,25-27)}$.

An important point to be emphasized refers to the difficulty in finding studies in the literature describing methods similar to those utilized in the present study. Most studies do not present any standardization pertinent to the sample characteristics, foods consistency, utensils and form of food offering. Thus, the present study results can provide a more critical view on the diagnosis of dysphagia in children, especially alerting speech therapists on the need to standardize the evaluation of such patients in the clinical practice, so as not to delay the adoption of approaches that are beneficial approaches to patients in terms of quality of life in cases where the presence of dysphagia is equivocally ruled out.

In such a case, an isolate clinical evaluation may be noneffective to detect aspiration with a given food consistency, which may result in health impairment in children with encephalopathy. On the other hand, aspiration is an intermittent phenomenon, as one test may demonstrate aspiration episodes while other may not ${ }^{(23)}$. Additionally, problems in the pharynx are more difficult to be perceived, a fact which justifies the difficulty in the diagnosis of changes in swallow.

The children who comprised the present study sample presented with heterogeneous motor, sensory and cognitive characteristics, a fact that may have affected the performance of the evaluations. However, the difficulty in classifying different types and manifestations of such neurological conditions is well known, as motor, cognitive, sensory and body global posture impairments are changes that are present in different degrees, making such population highly heterogeneous. Therefore, children with neurological disorders may present with changes in the dynamics of swallowing associated with compromised motor function and cognitive level, sensory changes or deprivation, regardless of the type or location of the lesion ${ }^{(1)}$.

Finally, it is important to highlight the role of the complementary investigation in the enhancement of the clinical diagnosis, as it promotes benefits to the patient, guiding the medical approach besides defining an individual therapeutic programming.

\section{CONCLUSION}

The clinical evaluation presented low sensitivity for the diagnosis of dysphagia with pureed consistency foods in children presenting with chronic encephalopathy of child- 
hood, as compared with videofluoroscopy. Therefore, the results of the present study demonstrate that the clinical diagnosis of dysphagia with the tested foods consistencies was enhanced by videofluoroscopy as a complementary investigation. Thus, the utilization of such method for complementary diagnosis should be assumed as an integral part of the protocol for evaluation of swallow changes, taking into consideration that the clinical evaluation must always precede any complementary investigation.

\section{REFERENCES}

1. Andrew MJ, Sullivan PB. Feeding difficulties in disabled children. J Paediatr Child Health. 2010;20:321-6.

2. Noll L, Rommel N, Davidson GP, et al. Pharyngeal flow interval: a novel impedance-based parameter correlating with aspiration. Neurogastroenterol Motil. 2011;23:551-e206.

3. Otapowicz D, Sobaniec W, Okurowska-Zawada B, et al. Dysphagia in children with infantile cerebral palsy. Adv Med Sci. 2010; 55:222-7.

4. Parkes J, Hill N, Platt MJ, et al. Oromotor dysfunction and communication impairments in children with cerebral palsy: a register study. Dev Med Child Neurol. 2010;52:1113-9.

5. Jayasekeran V, Singh S, Tyrrell P, et al. Adjunctive functional pharyngeal electrical stimulation reverses swallowing disability after brain lesions. Gastroenterology. 2010;138:1737-46.

6. DeMatteo C, Matovich D, Hjartarson A. Comparison of clinical and videofluoroscopic evaluation of children with feeding and swallowing difficulties. Dev Med Child Neurol. 2005;47:149-57.

7. Fung CW, Khong PL, To R, et al. Video-fluoroscopic study of swallowing in children with neurodevelopmental disorders. Pediatr Int. 2004;46:26-30

8. Low J, Wyles C, Wilkinson T, et al. The effect of compliance on clinical outcomes for patients with dysphagia on videofluoroscopy. Dysphagia. 2001;16:123-7.

9. Leder SB, Suiter DM, Green BG. Silent aspiration risk is volumedependent. Dysphagia. 2011;26:304-9.

10. Molfenter SM, Steele CM. Temporal variability in the deglutition literature. Dysphagia. 2012;27:162-77.

11. Erasmus CE, van Hulst K, Rotteveel JJ, et al. Clinical practice: swallowing problems in cerebral palsy. Eur J Pediatr. 2012;171:409-14.

12. McNair J, Reilly S. The pros and cons of videofluoroscopic assessment of swallowing in children. Asia Pacific Journal of Speech, Language and Hearing. 2003;8:93-104.

13. Chau KH, Kung CM. Patient dose during videofluoroscopy swal- lowing studies in a Hong Kong public hospital. Dysphagia. 2009; 24:387-90.

14. Omari TI, Papathanasopoulos A, Dejaeger E, et al. Reproducibility and agreement of pharyngeal automated impedance manometry with videofluoroscopy. Clin Gastroenterol Hepatol. 2011;9:862-7.

15. Osaki K, Kagaya H, Yokoyama M, et al. The risk of penetration or aspiration during videofluoroscopic examination of swallowing varies depending on food types. Tohoku J Exp Med. 2010;220:41-6.

16. Singh V, Berry S, Brockbank MJ, et al. Investigation of aspiration: milk nasendoscopy versus videofluoroscopy. Eur Arch Otorhinolaryngol. 2009;266:543-5.

17. Bossuyt PM, Reitsma JB, Bruns DE, et al. Towards complete and accurate reporting of studies of diagnostic accuracy: the STARD initiative. Standards for Reporting of Diagnostic Accuracy. Clin Chem. 2003;49:1-6.

18. Calis EA, Veugelers R, Sheppard JJ, et al. Dysphagia in children with severe generalized cerebral palsy and intellectual disability. Dev Med Child Neurol. 2008;50:625-30.

19. Trinick R, Johnston N, Dalzell AM, et al. Reflux aspiration in children with neurodisability - a significant problem, but can we measure it? J Pediatr Surg. 2012;47:291-8.

20. Shem K, Castillo K, Wong S, et al. Dysphagia in individuals with tetraplegia: incidence and risk factors. J Spinal Cord Med. 2011; 34:85-92.

21. McCullough GH, Wertz RT, Rosenbek JC. Sensitivity and specificity of clinical/bedside examination signs for detecting aspiration in adults subsequent to stroke. J Commun Disord. 2011;34:5572 .

22. Baylow HE, Goldfarb R, Taveira CH, et al. Accuracy of clinical judgment of the chin-down posture for dysphagia during the clini$\mathrm{cal} / \mathrm{bedside}$ assessment as corroborated by videofluoroscopy in adults with acute stroke. Dysphagia. 2009;24:423-33.

23. Baikie G, South MJ, Reddihough DS, et al. Agreement of aspiration tests using barium videofluoroscopy, salivagram, and milk scan in children with cerebral palsy. Dev Med Child Neurol. 2005;47:8693.

24. Rugiu MG. Role of videofluoroscopy in evaluation of neurologic dysphagia. Acta Otorhinolaryngol Ital. 2007;27:306-16.

25. Costa MMB. Videfluoroscopy: the gold standard exam for studying swallowing and its dysfunction. Arq Gastroenterol. 2010;47:3278.

26. Costa MMB. Videofluoroscopia: método radiológico indispensável para a prática médica. Radiol Bras. 2010;43(2):vii-viii.

27. Costa MMB, Canevaro LV, Azevedo ACP. Análise dosimétrica do método videofluoroscópico aplicado ao estudo da dinâmica da deglutição. Radiol Bras. 2000;33:353-7. 